\title{
La política habitacional después de los sismos
}

\author{
Priscilla Connolly*
}

El artículo tiene como objetivos analizar la acción gubernamental ante los daños habitacionales causados por los sismos y los efectos que de ésta se derivan sobre la política habitacional. El análisis se divide en cuatro etapas: diagnóstico del problema; el Programa Emergente de Vivienda, fase uno; el Programa de Renovación Habitacional Popular y la fase dos del Programa Emergente.

Por medio del análisis de las características del Programa de Renovación Habitacional Popular se identifican los contenidos programáticos innovadores, principalmente los que se refieren a los aspectos normativos de la construcción y planeación, y a las condiciones financieras. Sin embargo, se considera que la política habitacional general del país parece retomar las mismas tendencias que la han caracterizado.

Este artículo intenta responder a dos preguntas, distintas pero interrelacionadas. La primera de ellas es ¿cómo respondió el gobierno mexicano a los daños habitacionales causados por los sismos de septiembre de 1985?, y la segunda ¿qué efectos duraderos han tenido los sismos sobre la política habitacional? La respuesta a la primera interrogante la abordamos bajo la hipótesis de que los programas de reconstrucción en materia de vivienda fueron condicionados en gran medida por la política habitacional prevaleciente. Desde la percepción y cuantificación de los daños, hasta la puesta en marcha de proyectos definitivos de reconstrucción, la práctica estatal reprodujo los conceptos subyacentes a la política habitacional ejercida en las últimas décadas. Al mismo tiempo, y aquí entra en operación nuestra segunda hipótesis, los sismos, sus efectos y los programas habitacionales subsecuentes, seguramente contribuirán a transformar los concéptos que definen qué es y qué debe ser la política habitacional de este país.

1. Estimación de los daños habitacionales: un problema social y político

Los terremotos, a diferencia de desastres producidos por los hombres, como los bombardeos, raras veces son directamente dañinos a los seres humanos. Son las construcciones - edificios y obras diversas- las que matan, y su destrucción desquicia el funcionamiento normal de la sociedad. Las construcciones son artefactos producidos a partir de relaciones sociales; por esto, los efectos de los sismos no se deben a "causas naturales", sino que son hechos sociales. No sólo los daños producidos se determinan por

* UAM-Azcapotzalco. 
lo que existía antes, sino que también la identificación, descripción y evaluación de estos daños responden a concepciones, ideologías y objetivos políticos. No nos debe sorprender, entonces, un hecho que ha sido ampliamente comentado en relación con la tragedia de 1985: las incoherencias y amplias disparidades entre las diversas estimaciones, oficiales o no, de los daños, sobre todo en lo que se refiere a vivienda.

El cuadro 1 recordará al lector algunas de las versiones más notablemente dispares de la destrucción habitacional. Si lo contradictorio de las distintas versiones no nos sorprende, por lo menos merece algún comentario, que podemos referir a cuatro aspectos.

Como observación preliminar, llama la atención la enorme preponderancia de información referida a la ciudad de México, comparada con el resto del país. Ciertamente, en términos absolutos, fue en la capital y, especialmente, en los cuatro o cinco kilómetros cuadrados del centro, donde se produjo la mayor destrucción. Sin embargo, en términos relativos, y aún tomando como base la estimación máxima de 100000 viviendas afectadas en el Distrito Federal, los daños sufridos en Ciudad Guzmán y en sus municipios aledaños fue mucho mayor. En esta zona, se estimó la destrucción total de 1454 casas, más otras 1266 severamente dañadas, que en conjunto representan alrededor de $30 \%$ de todas las viviendas existentes en esa zona en el momento de los sismos (datos tomados de El Universal y La Jornada del 11 de octubre de 1985 y de Excélsior del 1 de diciembre de 1985). La poca atención prestada a los problemas habitacionales derivados de los terremotos fuera del área metropolitana de la ciudad de México (AMCM) y especialmente en las áreas rurales, empieza a comprobar nuestra primera hipótesis; tal hecho simplemente refleja la distribución geográfica, no sólo de la inversión pública sino también de las investigaciones en materia habitacional realizadas hasta la fecha. Lamentablemente, este ensayo no puede corregir ese sesgo, por lo que de aquí en adelante nos referiremos exclusivamente a la situación de la capital.

El segundo comentario va en el sentido siguiente: si el carácter y la magnitud de los daños se determinaron por las características de los edificios existentes, la estimación numérica también tuvo que ver con el conocimiento de la situación anterior. A pesar de que la ciudad de México y, sobre todo, el Distrito Federal, es con mucho la entidad más estudiada del país (y quizá se encuentre entre las ciudades más analizadas del mundo), la estadística disponible sobre la vivienda en ella es bastante escasa. No se sabe cuántos edificios o inmuebles hay en el AMCM, ni en el Distrito Federal; mucho menos se conoce la proporción que se destina a la vivienda. Ciertamente, se cuenta con estimaciones aproximadas acerca de los usos del suelo: $61 \%$ del área urbanizada en el Distrito Federal se destina a la habitación (DDF, 1984b), porcentaje ligeramente menor que la proporción de edificios habitacionales afectados por los sismos en relación con el total. Pero no se sabe cuántas viviendas se localizan en este 


\section{CUADRO 1}

Daños habitacionales y número de damnificados en el Distrito Federal, según diversas estimaciones

\begin{tabular}{|c|c|c|c|}
\hline Fecha & Fuente & $\begin{array}{l}\text { Viviendas o } \\
\text { familias }\end{array}$ & Personas \\
\hline \multicolumn{4}{|l|}{1985} \\
\hline $\begin{array}{l}27 \text { sept. } \\
7 \text { oct. }\end{array}$ & $\begin{array}{l}\text { CME (La Jornada) } \\
\text { Comisión Especial } \\
\text { Pluripartidista } \\
\text { (Proceso) }\end{array}$ & $\begin{array}{l}3600 \text { viviendas des- } \\
\text { truidas }\end{array}$ & $\begin{array}{l}40000 \text { damnificados } \\
30000 \text { damnificados }\end{array}$ \\
\hline 15 oct. & CEPAL (1er informe) & $\begin{array}{l}30000 \text { viviendas } \\
\text { destruidas y } 60000 \\
\text { viviendas dañadas }\end{array}$ & $\begin{array}{l}\text { más de } 150000 \text { dam- } \\
\text { nificados }\end{array}$ \\
\hline 17 oct. & $\begin{array}{l}\text { G. Carrillo A. } \\
\text { Sedue (La Jornada) }\end{array}$ & $\begin{array}{l}3500 \text { a } 4000 \text { fami- } \\
\text { lias damnificadas en } \\
\text { otras colonias }\end{array}$ & $\begin{array}{l}3200 \text { y } 750 \text { damnifi- } \\
\text { cados en Tlatelolco y } \\
\text { Unidad Benito Juárez, } \\
\text { respectivamente }\end{array}$ \\
\hline 19 oct. & CME (1er informe) & $\begin{array}{l}\text { carencia permanente } \\
\text { para no menos de } \\
13000 \text { familias }\end{array}$ & - \\
\hline 11 nov. & CUD (El Universal) & - & $\begin{array}{l}\text { aproximadamente } \\
250000 \text { damnificados }\end{array}$ \\
\hline 20 dic. & CME (Informe final) & $\begin{array}{l}3723 \text { edificios habi- } \\
\text { tacionales afectados } \\
\text { ( } 65 \% \text { del total) }\end{array}$ & $\begin{array}{l}\text { a proxima damente } \\
100000 \text { personas vi- } \\
\text { viendo en condicio- } \\
\text { nes precarias; } 37000 \\
\text { damnificados en al- } \\
\text { bergues y campamen- } \\
\text { tos }\end{array}$ \\
\hline \multicolumn{4}{|l|}{1986} \\
\hline 26 feb. & $\begin{array}{l}\text { Comité de Recons- } \\
\text { trucción para la } \\
\text { Zona Metropoli- } \\
\text { tana (Informe) }\end{array}$ & 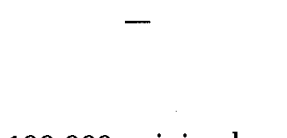 & $\begin{array}{l}54352 \text { damnificados } \\
\text { por pérdida total o } \\
\text { afectación severa de } \\
\text { su vivienda }\end{array}$ \\
\hline 18 sept. & $\begin{array}{l}\text { M. Camacho Solis } \\
\text { Sedue }\end{array}$ & $\begin{array}{l}100000 \text { viviendas } \\
\text { afectadas ( } 20000 \\
\text { familias sin aten- } \\
\text { derse en viviendas } \\
\text { sin daños severos) }\end{array}$ & \\
\hline
\end{tabular}

espacio. Extrapolando los datos censales para 1980, desglosados por municipio y delegación, se puede estimar que las 100000 viviendas afectadas representan alrededor de $25 \%$ de todo el acervo habitacional que existía en las delegaciones Venustiano Carranza y Cuauhtémoc, o $6 \%$ de todas las viviendas en el Distrito Federal. No obstante, los problemas relacionados con la captura de datos de ese censo impiden determinar la magnitud 
relativa de los daños habitacionales por localidades más reducidas (colonia u otro tipo de zona). Este desconocimiento con respecto al acervo habitacional de la ciudad de México constrasta con los registros existentes acerca del número, capacidad y ubicación de otros tipos de edificios afectados por los sismos, tales como las escuelas, los hospitales y las oficinas públicas. Si hubiera existido una información previa más precisa sobre la situación habitacional prevaleciente antes de los sismos, seguramente el panorama que se presentó de los daños sufridos habría sido muy distinto.

Otro factor relacionado con las limitaciones de los conocimientos previos acerca de la situación habitacional en la ciudad de México, también impidió la cuantificación precisa de los daños causados por los sismos a la vivienda. Este factor se refiere a la calificación del "daño". Excepto en los casos muy evidentes, como los derrumbes totales o parciales, ¿cómo y en qué medida se determina si una vivienda resultó dañada? Se podría pensar que una vivienda "afectada" por los sismos es aquella que se volvió inhabitable por esta causa. Pero aquí la cuantificación de los daños tropieza con los mismos problemas que los intentos de estimar los "déficit" habitacionales. ¿Qué se considera habitable y qué no? Según el estudio de la extinta coordinación general del Plan Nacional de Zonas Deprimidas y Grupos Marginales sobre necesidades esenciales, basado en datos censales de 1970, 81\% de las viviendas en el Distrito Federal (la entidad federativa con las mejores condiciones habitacionales) no cumplía con uno o varios de los criterios mínimos de habitabilidad; $11 \%$ de estas viviendas estaban totalmente deterioradas estructuralmente y otro $52 \%$ parcialmente deterioradas (Coplamar, 1982). Otras fuentes hablan de un déficit acumulado de aproximadamente 800000 viviendas en el Distrito Federal, o $45 \%$ del acérvo total de la entidad (DDF, 1984a). Así, hay discrepancias en cuanto a la definición de una vivienda "habitable", a la vez que, independientemente del criterio adoptado, una gran parte de las viviendas existentes antes de los sismos estaba inhabitable, un buen número de ellas, incluso, por su estado estructural ruinoso. Basta rccordar a los cuatro o cinco muertos que cada año reclamaba la época de lluvias, al desplomarse las podridas estructuras de adobe y bóveda de las antiguas vecindades centrales. Fue muy difícil, en estos casos, distinguir entre los estragos repentinos de los sismos y la destrucción acumulada durante años por el desgaste natural y la falta de mantenimiento.

Más allá de los problemas de información una cuarta consideración de orden social influyó sobre la manera de cuantificar los daños causados por los sismos. La cuantificación del problema dependió de lo que se pensaba hacer al respecto, sobre todo de los recursos a la vista para atenderlo. En otras palabras, el sapo fue del tamaño de la pedrada. Las versiones sucesivas de las estimaciones oficiales, entonces, iban cambiando de acuerdo con la evolución de las políticas de emergencia y reconstrucción habitacional. Es más, cifras contundentes y claras sólo se publicaron una vez 
que quedaron establecidos los programas de reconstrucción y, por supuesto, la magnitud de estas cifras siempre estuvo en relación con los alcances de los mismos. Por ejemplo, cuando la única respuesta clara del gobierno ante las necesidades de los damnificados era el ofrecimiento de un número limitado de créditos para obtener viviendas creadas por las instituciones financieras existentes, el cálculo oficial de les viviendas destruidas o irreparablemente dañadas fue de sólo 13300 . Casi un año después, cuando ya se conocía perfectamente la cantidad de acciones habitacionales terminadas o emprendidas por los diversos programas de reconstrucción, el titular de la Secretaría de Desarrollo Urbano y Ecología (Sedue) pudo anunciar que eran 100000 las familias cuyas viviendas fueron afectadas por los sismos (cuadro 1).

El alcance de la política de reconstrucción habitacional no sólo determinó la cuantificación oficial de los damnificados; también dictó la manera de describir y de clasificar a los mismos. A muy pocos días de la tragedia, los damnificados ya no fueron vistos como víctimas de un desastre, a quienes había que ayudar a toda costa, sino como beneficiarios potenciales de algún programa habitacional. En México, esto quiere decir ser sujeto de cualesquiera de los tipos de crédito otorgado por las diversas instituciones financieras de vivienda. Así, los damnificados empezaron a ser clasificados, de acuerdo con su elegibilidad para uno u otro tipo de crédito: notablemente por su capacidad de pago, pero también según su estatus de derechohabientes del Infonavit, el Fovissste o el Fovimi. Esta capacidad de tener acceso a los créditos otorgados por las instituciones habitacionales existentes también se sobrepuso, de una manera bastante burda, en el mapa de las zonas más afectadas, de acuerdo con los promedios de ingreso correspondiente a cada zona. De esta manera se clasificaron los damnificados. Los que vivían en las colonias llamadas "residenciales" o de "clase media", junto con los desalojados de la unidad NonoalcoTlatelolco y de la unidad habitacional Benito Juárez, por tratarse de personas solventes, podían sufragar los gastos normales de adquisición de una de las viviendas construidas en la periferia de la ciudad, que ya tenían en cartera los organismos financieros. En contraste, se reconoció ąue muy pocos de los habitantes de la zona anteriormente llamada "herradura de tugurios"', en su mayoría inquilinos en vecindades, podrían -o querríanacceder a un crédito para la adquisición de una de aquellas viviendas creadas por los programas convencionales. (Por lo demás, no había suficientes viviendas.) Pero ese sector de damnificados, el más numeroso por cierto, no fue identificado oficialmente -recuérdense las declaraciones del entonees titular de la Sedue- hasta que se convirtió en beneficiario de un programa habitacional, en este caso, hasta oue se tomó la medida excepcional de expropiar 5427 predios destinados al uso habitacional en el centro de la ciudad.

A pocos días de la publicación de la versión corregida del decreto 
expropiatorio, se acuñó la clasificación definitiva de los damnificados como "beneficiarios"; es decir, de acuerdo con su capacidad como sujetos de crédito. El primer desplegado del Programa Emergente de Vivienda, publicado en los periódicos de mayor circulación el 21 de octubre de 1985, dio a conocer la respuesta gubernamental a "la demanda de vivienda . . . de quienes la perdieron". Aquí se identifican claramente los tres grupos de "personas afectadas": el grupo "Tlatelolco-Unidad Benito Juárez"; el grupo "Roma, Juárez, Cuauhtémoc, Condesa y colonias aledañas" y, por último, el grupo "Barrios populares: raíces de nuestra ciudad". Días después, esta clasificación se reiteró en el primer informe de la Comisión Metropolitana de Emergencia y, de ahí en adelante, casi todos los estudios sobre los efectos de los sismos han adoptado el mismo esquema (véanse, por ejemplo, Ziccardi, 1986: 25, y Rodríguez, 1986: 43).

Quizá la manera de clasificar a los damnificados de acuerdo con el tipo de crédito que el habitante promedio de tal o cual zona pueda pagar, parezca lógica y natural en una sociedad clasista. De todas maneras, cabe reflexionar que en ningún momento se planteó la reconstrucción de hospitales, escuelas o edificios administrativos de acuerdo con la capacidad de pago de sus usuarios. Esto es porque el gasto público en el aprovisionamiento de tales elementos se considera claramente como un subsidio: un medio de redistribución del ingreso. No es así con la política habitacional en México. Aquí el Estado no regala vivienda o, más bien, no lo hace de manera explícita. Las inversiones en la habitación no se clasifican como gasto público, sino como préstamos recuperables, independientemente de si se amortizan o no, o en qué medida. De ahí que, en ningún momento (salvo cuando el donador especificara lo contrario) se contemplara la aplicación de recursos reunidos en el Fondo Nacional de Reconstrucción a la reconstrucción habitacional.

La filosofía financista de la política habitacional no sólo moldeó la atención pública a los damnificados después de los sismos. También influyó en las demandas de los propios damnificados. Un reiterado planteamiento de una de las organizaciones con más presencia, la de los habitantes de Tepito, fue que no esperaban regalos de nadie; sólo pedían ayuda para que ellos mismos resolvieran su problema. Posiblemente el lector de este artículo esté pensando en la imposibilidad de un enfoque alternativo para una política habitacional, diferente al criterio financista. ¿Cómo podía el gobierno mexicano regalar viviendas a un sector reducido de la población? ¿No sería totalmente injusto que sólo unos pocos fueran subvencionados con fondos públicos o con los donativos? En las secciones siguientes se intentará demostrar, entre otras cosas, que ni los programas habitacionales existentes que se aplicaron en la primera fase del Programa Emergente, ni los programas creados expresamente en atención a los damnificados eliminaron el subsidio, sino que, por el contrario, el nivel de subvención en todos los casos fue altísimo; y que estas subvenciones, como 
casi todos los efectos de la política social en México, benefician a un sector muy limitado y circunscrito de la población.

2. La aplicación de los programas habitacionales existentes: el Programa Emergente de Vivienda, fase uno

La primera respuesta del gobierno mexicano ante la destrucción habitacional causada por los sismos fue congelar las asignaciones existentes de la vivienda en cartera de los diversos organismos públicos financieros, para canalizarla en atención a los damnificados. En una reunión convocada por la Comisión Metropolitana de Emergencia el 30 de septiembre, los titulares de estos organismos manifestaron la disponibilidad de viviendas terminadas o de próxima terminación en el AMCM (columna izquierda del cuadro 2). Las unidades disponibles en este primer recuento sumaron 14 146, cifra no muy diferente al número acumulado de viviendas entregadas por el llamado Programa Emergente de Vivienda hasta septiembre de 1986 (véase el cuadro 3).

El Programa Emergente fue creado para atender a sectores especificos y limitados de los damnificados: en primer término, a la población residente de las dos unidades habitacionales -Benito Juárez y NonoalcoTlatelolco- cuya destrucción implicaba responsabilidades directas de organismos estatales: el ISSSTE y Fonhapo-Banobras, respectivamente. En ambos casos hubo derrumbes espectaculares, con aproximadamente 750 muertos y 350 desaparecidos sólo en Tlatelolco. En este conjunto habitacional los antecedentes de organización política entre los residentes propiciaron, además, una pronta movilización para demandar no sólo las soluciones y compensaciones adecuadas al caso, sino también el deslinde de responsabilidades y el enjuiciamiento de los culpables. ${ }^{1}$

${ }^{1}$ La situación suscitada en la unidad Nonoalco-Tlatelolco a raíz de los sismos es sumamente compleja, tanto por la magnitud del conjunto habitacional (11 906 departamentos en 102 edificios), como por la heterogeneidad de los afectados (se puede identificar a por lo menos seis grupos, con intereses y derechos distintos: propietarios residentes, propietarios arrendadores, inquilinos, subarrendadores, inquilinos de los edificios administrados por el ISSSTE y habitantes de los cuartos de azotea). La población residente de una parte de los edificios estaba organizada para la autoadministración de los mismos, pero no todos estaban bajo este régimen. Además, no todos los edificios sufrieron el mismo grado de daños, ya que éstos van desde alteraciones insignificantes hasta la destrucción total. Las acciones del Estado en este caso incluyen la resolución de los pagos de indemnizaciones a deudos y propietarios de los departamentos destruidos, la definición del grado de afectación de los edificios, la propuesta correspondiente de un proyecto de reconstrucción de la unidad, así como la asignación de créditos habitacionales a los afectados que acudieron al Programa Emergente de Vivienda. El análisis de todos estos procesos desborda evidentemente los alcances del presente trabajo, por lo que nos limitamos al aspecto de la participación de los afectados de esta unidad habitacional en el Programa Emergente. 


\section{CUADRO 2}

Programa Emergente de Vivienda, fase uno: disponibilidades y asignaciones por organismo

\begin{tabular}{|c|c|c|c|c|}
\hline Organismo & $\begin{array}{c}\text { Número de } \\
\text { viviendas } \\
\text { disponibles } \\
\text { en septiembre } \\
\text { de } 1985 \text { en la } \\
\text { ZMCM }^{1}\end{array}$ & $\begin{array}{c}\text { Número de } \\
\text { cédulas } \\
\text { canalizadas } \\
\text { por Sedue } \\
\text { al } 20 / 1 / 86^{2}\end{array}$ & $\begin{array}{c}\text { Número de } \\
\text { viviendas } \\
\text { asignadas } \\
\text { al } 20 / 1 / 86^{2}\end{array}$ & $\begin{array}{c}\text { Número de } \\
\text { créditos } \\
\text { asignados } \\
\text { al } 20 / 1 / 86^{2}\end{array}$ \\
\hline \multirow[t]{2}{*}{ Fovi / banca } & 3945 & 2229 & 661 & - \\
\hline & $30 \%$ & $16 \%$ & $13 \%$ & - \\
\hline \multirow[t]{2}{*}{ AURIS } & 1620 & 1623 & 490 & - \\
\hline & $11 \%$ & $11 \%$ & $9 \%$ & - \\
\hline \multirow[t]{2}{*}{ Fovissste } & 2704 & 5747 & 1541 & $4234^{*}$ \\
\hline & $19 \%$ & $41 \%$ & $30 \%$ & \\
\hline \multirow[t]{2}{*}{ Infonavit } & 5618 & 4072 & 2347 & 332 \\
\hline & $40 \%$ & $29 \%$ & $45 \%$ & \\
\hline \multirow[t]{2}{*}{ Pemex } & n.d. & 339 & 145 & 50 \\
\hline & - & $2 \%$ & $3 \%$ & \\
\hline \multirow[t]{2}{*}{ CFE } & n.d. & 43 & 24 & 12 \\
\hline & - & $0.3 \%$ & $0.5 \%$ & \\
\hline \multirow[t]{2}{*}{ Fovimi / Issfam } & n.d. & 30 & n.d. & n.d. \\
\hline & - & $0.2 \%$ & - & \\
\hline \multirow[t]{2}{*}{ Fonhapo } & 160 & 0 & $\mathbf{0}$ & $\mathbf{0}$ \\
\hline & $1 \%$ & - & - & \\
\hline \multirow[t]{2}{*}{ Total } & 14146 & 14083 & 5208 & 4628 \\
\hline & $100 \%$ & $100 \%$ & $100 \%$ & \\
\hline
\end{tabular}

Fuentes: ${ }^{1}$ Sedue, Subsecretaría de Vivienda.

${ }^{2}$ Sedue, Subsecretaría de Vivienda; datos reproducidos en Ziccardi (1986: 54).

* Incluye 1365 créditos otorgados directamente por el Fovissste para reparaciones de vivienda. n.d. no disponible.

El otro sector de damnificados identificados como beneficiarios potenciales del Programa Emergente de Vivienda, curiosamente llamado "población abierta", corresponde a familias que perdieron sus viviendas en las colonias consideradas "de clase media", en virtud de lo cual se presumía que tales familias pudiesen ser sujetos de crédito para alguno de los organismos financieros de vivienda.

La estimación inicial del número de unidades necesarias para realojar a los sectores antes mencionados fue de 8 756: 3020 de Tlatelolco, 736 de la unidad habitacional Benito Juárez y 5000 de las colonias medias. Por lo anterior, las 14146 viviendas disponibles se consideraron suficientes para cubrir esta necesidad. Sin embargo, tal estimación falló por varias razones. En primer lugar, el número de damnificados en Tlatelolco y, sobre todo, la demanda de la llamada "población abierta", fueron mucho mayores de lo esperado. De hecho, las solicitudes de atención desbordaron la deli- 
LA POLÍTICA HABITACIONAL DESPUÉS DE LOS SISMOS

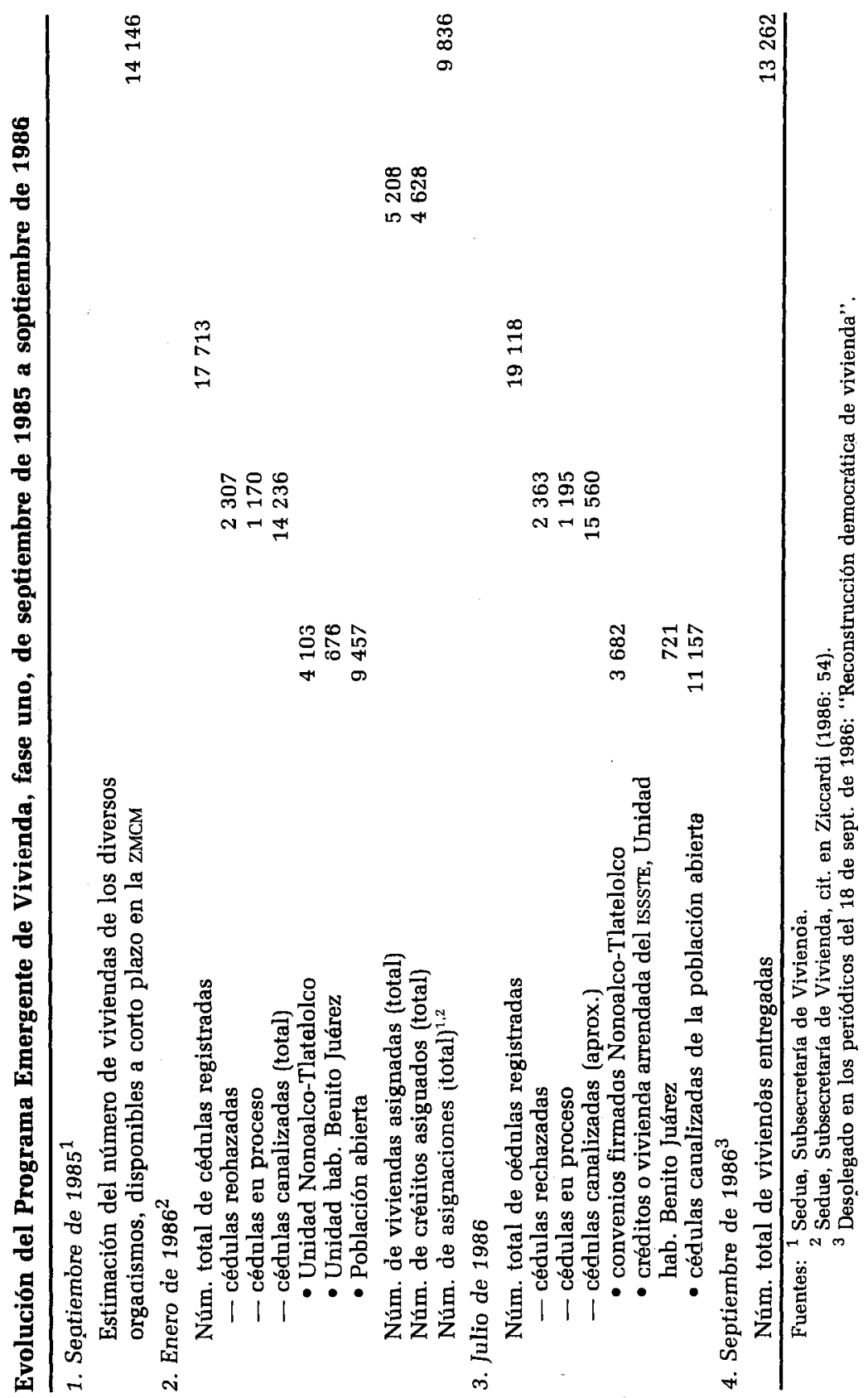


mitación territorial originalmente identificada, correspondiente principalmente a la delegación Cuauhtémoc; $35 \%$ de ellas provino de otras nueve delegaciones e, incluso, del Estado de México. Lo anterior ocurrió a pesar de la apertura del programa a familias afectadas por la expropiación de predios en las colonias populares que, siendo derechohabientes o sujetos de crédito de los organismos viviendistas, optasen por adquirir una casa o departamento ofrecido por el Programa Emergente en lugar de beneficiarse del Programa de Renovación Habitacional Popular.

A pesar de la ampliación del universo territorial del Programa Emergente -o quizá a causa de ello-, muchos damnificados en las,colonias donde operaba el programa no fueron beneficiados del mismo. Entre otras cosas, la vivienda ofrecida se ubicaba en las afueras de la ciudad y generalmente no igualaba ni la calidad ni el tamaño de los departamentos y casas destruidos por el temblor. Asimismo, gran parte de la supuesta población beneficiaria del programa no resultó ser tan económicamente solvente como se pensaba: poco más de tres cuartas partes de los damnificados atendidos por el Programa Emergente declararon percibir ingresos familiares de menos de cuatro veces el salario mínimo, límite mínimo para acceder a créditos habitacionales de la banca privada o de AURIS, por ejemplo (datos de Sedue citados en Ziccardi, 1986 y en Fonhapo, 1986). Este hecho explica las diferencias entre la participación relativa de los distintos organismos en el programa, originalmente y en su aplicación final, sobre todo, la menor importancia del Fovi y AURIS (véase el cuadro 2).

Sólo un grupo de este "sector territorial" de damnificados, frente a su evidente incapacidad para acceder a una vivienda del Fovi y su ineligibilidad para beneficiarse de los demás organismos habitacionales, logró negociar una solución alternativa a los créditos ofrecidos por el Programa Emergente. Los residentes de los cuartos de azotea de la unidad Nonoalco-Tlatelolco, organizados en su unión correspondiente, fueron incluidos como beneficiarios del programa de Renovación Habitacional Popular.

Los requisitos de ingresos para acceder a un crédito habitacional de Fovi o AURIS, no fueron las únicas condiciones impuestas a los damnificiados para beneficiarse del Programa. Los demás organismos financieros atendían sólo a sus derechohabientes y, en este sentido, igual que en la determinación de las condiciones del préstamo, se presentaron escasas diferencias con respecto a sus reglas de operación normales. Desde el punto de vista de los beneficiarios, su condición de damnificado sólo significó dos concesiones en cuanto al costo de su nueva vivienda. A los adquirientes de casas o departamentos financiados por la banca, se les concedió una reducción del enganche y mayores facilidades para el pago del mismo. En segundo lugar, a todos los beneficiarios del programa y por una sola vez, se les eximió del pago de los gastos relacionados con la compra de su vivienda: del impuesto sobre la adquisición de inmuebles y de 
los gastos notariales. La exención del impuesto sobre la adquisición de inmuebles no era del todo excepcional, ya que existía un antecedente para los compradores de vivienda de los organismos públicos en la Ley de Hacienda para el DDF vigente en 1982: exención que no fue incluida en las versiones posteriores de esta ley, promulgadas después de diciembre de ese año.

Por lo demás y desde el punto de vista del organismo financiero, las condiciones crediticias aplicadas al Programa Emergente fueron las normales, es decir, implicaron el mismo nivel de subsidio que de costumbre. A pesar de las dificultades económicas que los beneficiarios pudieran tener para cubrir los pagos de amortización del préstamo, las condiciones de éste implican un subsidio muy alto. En el caso del Programa Financiero de Fovi y la banca, este subsidio lo constituye la diferencia entre la tasa de interés pagada por el adquiriente de la vivienda $-35 \%$ aproximadamente- y la tasa bancaria comercial de más de $100 \%$. En el caso de los demás organismos, el subsidio es bastante mayor. Un beneficiario de un préstamo del Infonavit, por ejemplo, sin haber desembolsado enganche alguno, paga $4 \%$ de interés anual sobre los saldos insolutos de un préstamo equivalente al precio inicial de una vivienda que, en la situación inflacionaria actual, duplica su valor comercial cada año. Las condiciones del Fovissste son similares, salvo que la calidad y precio de la vivienda generalmente son mayores y, en el caso de la Comisión Federal de Electricidad, no se cobran ni enganche ni intereses sobre el préstamo habitacional. Bajo estas condiciones, no sorprende el hecho de que el Infonavit no logre dotar de vivienda anualmente a más de $1.8 \%$ de sus derechohabientes, y que este porcentaje se reduzca a menos de $1 \%$ en el caso del Fovissste (datos del Programa Nacional para el Desarrollo de la Vivienda para 1985).

Si el Programa Emergente de Vivienda no introdujo novedad alguna con respecto a sus condiciones financieras y de las características y ubicación de la vivienda ofrecida a los damnificados, hay un aspecto de su ejecución que constituye un cambio importante en el ejercicio de la política habitacional estatal. Este punto ya ha sido comentado por Ziccardi (1986: 52) y se refiere a la centralización del control general del programa, así como del primer nivel de reconocimiento de los beneficiarios, en manos de la Sedue. En efecto, el primer interlocutor de la población damnificada fue esta secretaría, en la forma de los cuatro módulos que la misma estableció en lugares estratégicos de la ciudad. En estos módulos, la población afectada debió comprobar su calidad de damnificado y proporcionar los datos socio-económicos necesarios, para que luego su solicitud se canalizara al organismo apropiado. Así, la Sedue asumió la responsabilidad global de la atención de los damnificados y se relativizó el poder independiente de los organismos. Esta tendencia centralizadora es especialmente clara en relación con el caso de la unidad Nonoalco-Tlatelolco. Aquí, 
a pocos días de los sismos, la Sedue asumió la plena responsabilidad para resolver la situación, tanto en lo referente al pago de indemnizaciones a deudos y a los propietarios de los departamentos dañados, como a los dictámenes técnicos, la determinación de los programas de reconstrucción de la unidad y la integración de los expedientes de los damnificados para su canalización posterior a los organismos financieros apropiados.

Los organismos financieros de vivienda, sin embargo, no perdieron del todo su capacidad de acción autónoma, hecho que podemos constatar al comparar el comportamiento diferencial del Infonavit y el Fovissste después de los mismos. El titular del Infonavit se mostraba renuente a cancelar las asignaciones de vivienda ya comprometidas con los sindicatos promotores, y se negó rotundamente a poner a la disposición de damnificados no derechohabientes de su organización las viviendas terminadas en cartera (véase Proceso del 14 de octubre de 1985). Esta posición contrasta con la del Fovissste, que no sólo aportó al Programa Emergente la totalidad de la vivienda en cartera, sino también puso en marcha de inmediato programas propios de atención a los damnificados en la forma de líneas de crédito para reparaciones. La mayor preocupación del Fovissste con las consecuencias del terremoto puede explicarse, en parte, por el involucramiento directo del ISSSTE en los casos de las unidades habitacionales Benito Juárez y parte de Tlatelolco, así como por la alta proporción de servidores públicos, derechohabientes del organismo, entre los residentes del área central de la ciudad de México.

En resumen, el Programa Emergente de Vivienda permitió resolver una parte de las necesidades habitacionales derivadas de los sismos, sin mayores reajustes políticos, programáticos o presupuestales. Desde luego, esta fórmula sólo pudo resolver una mínima parte del problema; excluyó a los damnificados que en virtud de sus ingresos, situación ocupacional, condición familiar u otra causa, no cumpliesen con los requisitos acostumbrados para acceder ni a una vivienda financiada por el sector público ni, de hecho, a cualquier vivienda considerada "digna" o adecuada. La atención a estos damnificados - a los damnificados de siempre-constituyó el verdadero reto de la reconstrucción habitacional. Por un lado, el rechazo político del subsidio habitacional impidió destinar recursos donados al Fondo Nacional de Reconstrucción hacia el aprovisionamiento de vivienda para los damnificados: la solución tenía que comprender mecanismos de recuperación. Por otro, los fondos disponibles eran reducidos en relación con la magnitud del problema.

Ante la doble carencia de medios económicos e instrumentales, el Estado mexicano hizo valer un tercer recurso gratuito pero reservado para contingencias especiales: su prerrogativa constitucional de imponer a la propiedad privada las modalidades que dicte el interés público, mediante la expropiación. Sin duda, la expropiación masiva de predios ocupados por vivienda multifamiliar de bajo costo en los llamados "barrios popula- 
res"' en el centro de la ciudad de México constituye el elemento clave del programa de reconstrucción Renovación Habitacional Popular. Un análisis de los antecedentes, instrumentación jurídica y efectos de la expropiación se presenta en el artículo de A. Azuela en este volumen. Igualmente, los aspectos coyunturales, operacionales y los efectos del propio Programa de Renovación Habitacional Popular, en lo que se refiere a los actores sociales e institucionales, están tratados en el trabajo de E. Duhau. En los párrafos que siguen, no es nuestra intención duplicar estos aportes analíticos e informativos, sino identificar los aspectos del programa que son innovadores y que constituyen pautas duraderas para el desarrollo de la política habitacional al nivel nacional e, incluso, internacional.

3. "Raíces de nuestra ciudad": el programa

Renovación Habitacional Popular

A primera vista, son muchos los contenidos programáticos novedosos introducidos por Renovación Habitacional Popular. Un examen más cuidadoso puede revelar no sólo lo efímero de algunos de sus componentes sino, en muchos aspectos, la reproducción de esquemas característicos de la política habitacional en este país desde hace mucho tiempo.

Probablemente la característica más excepcional de Renovación Habitacional Popular son sus propios objetivos generales. Tanto en los causales de la expropiación estipulados en octubre de 1985 (véase Azuela, 1987: 6-7), como en los objetivos y estrategias del programa definitivo redactado siete meses después (RHP, 1986: 3-4), se expresa claramente la intención de reconstruir viviendas en beneficio de los habitantes originales, arraigando así a la población de menores recursos en el centro de la ciudad. A nivel retórico, tal objetivo político no representa novedad alguna. La experiencia tanto nacional como extranjera puede demostrar que se han justificado con argumentos y objetivos semejantes casi todos los programas públicos de "renovación urbana". Pero éstos, fundamentados igualmente en la expropiación y fusión de la propiedad fragmentada de los predios, generalmente han tenido la connotación de "acción bulldozer", la expulsión de los pobres, la construcción para usos más rentables y el negocio inmobiliario. Tales programas suelen aplicarse en momentos de auge económico, cuando las tasas de interés están bajas y los precios del suelo en su apogeo. Aunque algunos autores afirman lo contrario (López Monjardin, 1984: 27), estas condiciones propicias para el "empuje de los procesos especulativos" no se presentaban en México en octubre de 1985. Por consiguiente, ni la expropiación ni el programa de renovación subsecuente pueden compararse con los proyectos acostumbrados de "renovación urbana"; podemos aceptar que sus objetivos sean sinceros.

Los objetivos dé Renovación Habitacional Popular no son excep- 
clonales sólo por sus buenas intenciones; más sorprendente todavía es que se estén cumpliendo en la práctica. Se puede constatar que los beneficiarios de los recién estrenados departamentitos y accesorias construidos por el programa son, en efecto, las mismas familias que ocupaban las viviendas y locales comerciales existentes anteriormente en el predio. Ciertamente, no faltan antecedentes de programas de sustitución de vivienda deteriorada en beneficio de su población original, incluso en el área central de la ciudad de México (véase Coulomb, 1985). Pero generalmente estas experiencias se han desarrollado a una escala sumamente modesta (la Cooperativa colonia Guerrero, por ejemplo) o no han logrado cabalmente el objetivo de beneficiar a los habitantes originales (el programa de Banobras de sustitución de vecindades y aun el Plan Tepito). Así, como programa masivo de reconstrucción de viviendas que efectivamente se destinen a los habitantes originales del área central de una gran ciudad, el programa Renovación Habitacional Popular quizá no tiene precedentes y no sólo en México, sino también en el mundo.

\section{CUADRO 4}

\section{Programa Renovación Habitacional Popular:} metas y avance a noviembre de 1986

\begin{tabular}{lrrr}
\hline & $\begin{array}{c}\text { Metas } \\
\text { junio de 1986 }\end{array}$ & $\begin{array}{c}\text { Metas } \\
\text { noviembre de 1986 }\end{array}$ & $\begin{array}{c}\text { Viviendas } \\
\text { terminadas } \\
\text { (15 de noviembre } \\
\text { de 1986) }\end{array}$ \\
\hline $\begin{array}{l}\text { Número de viviendas } \\
\quad \text { Total }\end{array}$ & 44437 & 42000 & 8729 \\
$\begin{array}{l}\text { Demolición y obra } \\
\text { nueva }\end{array}$ & 28302 & 34500 & 6413 \\
$\begin{array}{l}\text { Rehabilitación } \\
\text { Reparaciones menores }\end{array}$ & 11649 & 6700 & 1748 \\
Vivienda provisional & 4486 & 800 & 568 \\
Ayuda de renta & 26007 & n.d. & 13093 \\
\hline
\end{tabular}

Fuente: Renovación Habitacional Popular.

n.d., no disponible

Si Renovación Habitacional Popular está cumpliendo sus objetivos generales en cuanto a la población beneficiaria, los resultados hasta la fecha no dejan de presentar algunas discrepancias en relación con las metas planteadas. El cuadro 4 demuestra los alcances del programa y el avance del mismo a finales de 1986; en él se puede constatar que, a pesar de la eliminación del programa de un buen número de predios a causa de juicios de amparo favorables a los propietarios, las metas revisadas sólo han bajado en 2437 viviendas. Donde sí hay discrepancias entre el programa origi- 
nal y el revisado es en el avance; hasta noviembre de 1986, se había logrado terminar la construcción de poco menos de la mitad de las 18332 viviendas programadas para el fin de año. Tampoco se logró construir la cantidad programada de viviendas provisionales, condición fundamental para la realización del proyecto definitivo.

Todavía se registran mayores ajustes en la distribución programática de las acciones. Uno de los aspectos innovadores del programa -en su planteamiento original - fue la inclusión de obras de rehabilitación y de reparaciones menores: correspondientes a aproximadamente 25 y 10 por ciento del programa total respectivamente. En la versión revisada del programa estos porcentajes se reducen a 16 y 2 por ciento. La modificación posiblemente se explica por la falta de experiencia en el manejo de este tipo de proyectos, así como por errores de juicio en los dictámenes técnicos correspondientes. Por otro lado, es conocida la preferencia de las constructoras, agentes fundamentales en la ejecución del programa, por hacer contratos de obra nueva sobre rehabilitaciones, lo que puede haber conducido a demoliciones precipitadas en algunos casos. También se sabe que, en los predios donde existen convenios con el INAH y el INBA, los procedimientos se han complicado, entre otras cosas por los elevados costos de la restauración de los edificios. Por último, y por lo que se ha podido observar en las obras entregadas, la distinción entre "rehabilitación" y "reparaciones menores" es insignificante, es decir, probablemente, la "rehabilitación" mayor está resultando demasiado costosa e impráctica como solución de mejoramiento habitacional en la ciudad de México.

Una última discrepancia observable entre las dos versiones de Renovación Habitacional Popular se refiere a la distribución geográfica de las acciones. Los cambios más notables en este sentido se ubican en Tepito, donde se construirán casi el doble de las viviendas originalmente programadas, en la zona Centro-norte y Merced-Tomatlán, donde el número de acciones es ligeramente mayor en la nueva programación, y en la "zona 6", es decir, las colonias Atlampa, Vallejo poniente, Peralvillo y Felipe Pescador, donde se ha reducido considerablemente el número de acciones programadas. Se desconocen las razones de estas modificaciones; pueden haber influido desde errores técnicos originales hasta la distribución geográfica de los predios amparados contra la expropiación. No habrá que descartar la posible injerencia de las presiones ejercidas por los residentes de las colonias respectivas.

Independientemente de los objetivos y alcances del programa y el grado de cumplimiento de los mismos, quizá las innovaciones más interesantes de Renovación Habitacional Popular residen en sus aspectos operacionales. Aparte de la envergadura de las acciones, ¿qué factores le distinguen de otros proyectos con fines similares? y ¿que características del programa explican su relativo éxito en la consecución de los objetivos generales planteados? A nuestro juicio, son dos los factores fundamenta- 
les que explican la viabilidad del programa: el aspecto normativo de los proyectos y las condiciones financieras. Estos dos factores se conjugan en la determinación de un tercer aspecto, el más importante en la práctica: la administración del programa desde el punto de vista de su organización social y técnica.

Las innovaciones de orden normativo estriban en el hecho de que casi todo el marco reglamentario referido a la construcción y planeación urbana se hizo a un lado. En su lugar, se contrató el diseño de 11 proyectos prototipo, de acuerdo con un conjunto de normas elaboradas (también bajo contrato) ex profeso para la organización Renovación Habitacional Popular. La implicación principal de la no adherencia a los reglamentos existentes -mismos que, por lo demás, habían demostrado su poca eficacia en los sismos-, fue la posibilidad de construir a densidades mucho mayores que lo normalmente permitido. En especial, se eliminaron los requisitos que habían constituido los principales obstáculos para la realización de proyectos de "sustitución de vecindades" en el pasado: el aprovisionamiento de un cajón de estacionamiento por vivienda y la donación de espacios abiertos o la realización de pagos compensatorios para tal efecto. Si se hubiese cumplido con estos requisitos habría sido imposible realojar a todas las familias en los mismos predios, y mucho menos, como en algunos casos, aumentar su densidad. Por otra parte, la construcción de prototipos habitacionales de $40 \mathrm{~m}^{2}$ de superficie construida facilitó la operación técnica del programa.

La flexibilidad normativa de los proyectos permitió (o fue indispensable para) la aplicación de un criterio urbanístico inusitado en un programa de renovación de esta envergadura: la conservación estricta de la lotificación anterior. La reconstrucción de más o menos el mismo número de viviendas, con las mismas familias en el mismo predio, pudo haber resultado de un deseo de conservar "la vecindad", tan alabada por todos los sectores en las discusiones que tuvieron lugar en los meses posteriores a los sismos. Sin embargo, las características arquitectónicas de los prototipos de Renovación rescatan poco de aquella fórmula tradicional de vivienda colectiva; más bien emulan el departamento convencional a una escala reducida. Lo significativo de la conservación del predio como unidad base, tanto para la siembra de los prototipos como para la constitución de la propiedad en "condominio vecinal", reside entonces en el nivel de la administración del programa; los beneficiarios están organizados por predio. Si esta solución parece obvia por la génesis del programa, no deja de ser innovadora y excepcional, con implicaciones importantes y, quizá, duraderas para la configuración social de los residentes del área central. Para un análisis de las implicaciones del "condominio vecinal", véase el artículo de $\mathrm{E}$. Duhau en este volumen.

La última faceta del programa Renovación Habitacional Popular que, aparentemente, ofrece parámetros nuevos, es el aspecto financiero. Con 
la excepción de un proyecto de autoconstrucción cerca de Ciudad Lázaro Cárdenas, Michoacán, durante la década pasada, y a diferencia de muchos otros países del tercer mundo, México no había recibido financiamientos del Banco Mundial para programas habitacionales. Así, al parecer, Renovación Habitacional Popular estrena una nueva tendencia en la política habitacional mexicana: la de ser sujeto de créditos externos negociados bilateralmente con el Banco Internacional de Reconstrucción y Fomento (BIRF), filial del Banco Mundial. Sin embargo, tal como señala Duhau (1987: 23-24), más de la tercera parte de este financiamiento corresponde a la reorientación de un préstamo ya concertado por Fonhapo con el BIRF, para programas de vivienda en la zona del Golfo e Istmo. Es decir, de todas maneras México iba a hacer su debut entre los clientes del Banco Mundial en materia de financiamiento habitacional. Una de las implicaciones principales de este arreglo con el BIRF es la reducción —otra vez aparentedel nivel de subsidio permitido no sólo en la propia Renovación Habitacional Popular sino también en todas las líneas operacionales del Fonhapo.

El calificativo "aparente" aplicado a la reducción del subsidio exigida por el BIRF se refiere a las ambigüedades implícitas en la manera de estimar el subsidio. El financiamiento externo, equivalente a aproximadamente 97 mil millones de pesos, sólo se destina a los gastos en la construcción o reparación de las viviendas, junto con otros 28.6 mil millones de recursos fiscales aportados al programa. Esta parte del financiamiento total del programa constituye el gasto supuestamente recuperable, de acuerdo con los límites de subsidio impuesto por el Banco Mundial. Más de la tercera parte del gasto total programado para el proyecto, $74.5 \mathrm{mil}$ millones de pesos, corresponde a recursos fiscales adicionales considerados como "fondo perdido". Los rubros más importantes de esta parte del gasto son el impuesto al valor agregado, la construcción de vivienda provisional, las demoliciones, la supervisión de obra y la realización de estudios y proyectos así como los gastos corrientes del programa. Las erogaciones en todos estos conceptos, entre otros menores, representan entonces un subsidio directo y a $\mathbf{1 0 0 \%}$ a los beneficiarios del programa. Si este subsidio resulta mayor que el otorgado a los adquirientes de una vivienda del Infonavit, por ejemplo, resulta difícil de estimar, sobre todo porque los costos reales de la vivienda construida o rehabilitada por Renovación Habitacional Popular todavía se desconocen. En efecto, aunque el monto de los pagos mensuales para el rembolso del crédito está acordado tanto en el Convenio de Concertación Democrática como en los contratos individuales de compra-venta entre los beneficiarios y Renovación Habitacional Popular, nadie sabe cuál es el monto total del cŕedito. Evidentemente, los precios anitarios utilizados para presupuestar las bases deI programa tienen poco que ver con los precios de nroducción reales, dadas la alta tasa de inflación y erogaciones imprevistas. Difícilmente se puede determinar el grado de subsidio sin conocer este costo real de programa. 
Sea como sea, el nivel de subsidio otorgado por Renovación Habitacional Popular es bastante alto, tan alto que podemos pensar que las viviendas son un virtual regalo. Lo anterior es cierto no obstante las condiciones de recuperación impuestas por el Banco Mundial y no obstante las dificultades que algunos de los beneficiarios puedan tener para enfrentar las mensualidades, sobre todo porque muchos de ellos no están acostumbrados a pagar más que "rentas congeladas". En este sentido, el programa no sale de la pauta histórica característica de la política habitacional mexicana: la de canalizar una alta concentración de recursos económicos, sociales y políticos en beneficio, por una sola vez, de un sector específico y limitado de la población. En este sentido, también, cabría preguntar si Renovación Habitacional Popular no hace más que reproducir la esencia del régimen de "rentas congeladas" que pretendió sustituir.

\section{4. "Fase dos": el regreso a la normalidad}

Quizá la prueba más inmediata del carácter único y exclusivo de Renovación Habitacional Popular pueda encontrarse en el Programa Emergente de Vivienda, fase dos. Convocado en agosto de 1986, en abierto reconocimiento de las fallas e insuficiencias de Renovación Habitacional Popular, el "fase dos" pretende ofrecer una solución a aquellos inquilinos cuyas viviendas fueron afectadas por los sismos pero que no se incluyeron en la expropiación. Así, en lugar de ampliar o repetir el decreto expropiatorio, tal como lo demandaban las principales organizaciones de damnificados, se optó por introducir un nuevo programa, cuyas modalidades de operación tienen muçho más que ver con los sistemas convencionales de financiamiento habitacional, especialmente el del Fonhapo, que con Renovación Habitacional Popular.

La primera diferencia importante entre los dos programas se refiere a la relación entre el organismo responsable y los beneficiarios. Los residentes de predios expropiados realmente no tuvieron otra opción más que incorporarse a Renovación Habitacional Popular y, en casi todos los casos, el acercamiento entre vecinos y el organismo tuvo lugar a instancias de éste. Por el contrario, "fase dos" convocó a los inquilinos interesados a solicitar su inscripción en el programa; la concurrencia al mismo fue por iniciativa de los beneficiarios. Por lo tanto, requirió de un grado considerablemente mayor de organización previa de parte de ellos. Inclusive, en algunos casos, la procedencia del proyecto dependió (y sigue dependiendo) del grado de combatividad de los futuros beneficiarios para, entre otras cosas, negociar la venta del terreno con el propietario.

En segundo término, las condiciones financieras y crediticias ofrecidas por "fase dos" nada tienen que ver con la generosidad absoluta de Renovación Habitacional Popular. Los beneficiarios no sólo pagarán el 
costo del suelo, cuyo monto se incluirá en los pagos mensuales de amortización; tendrán que desembolsar, además, un enganche equivalente a $10 \%$ del costo de la obra. ${ }^{2}$

En tercer lugar, a los beneficiarios de "fase dos" se les exigieron una serie de requisitos, como comprobar que su vivienda quedó afectada seriamente por el temblor, comprobar ingresos mensuales menores a 2.5 veces el salario mínimo, etc. Todo parece indicar el regreso al manejo burocrático de los programas habitacionales.

Por último, los alcances de 'fase dos' son bastante modestos tanto en relación con los programas emergentes anteriores como en cuanto al tamaño del problema. A mediados de noviembre de 1986, "fase dos" contemplaba la construcción de 12590 viviendas y la rehabilitación de otras 3350 , y el avance fue de aproximadamente 6000 viviendas en 283 predios. Para esas fechas se había cerrado la convocatoria para las solicitudes de crédito, aunque según las declaraciones del director del programa "si el edificio resultó dañado, 'fase dos' puede atender el problema todavía".

Teóricamente, la primera fase del Programa Emergente de Vivienda ya terminó; Renovación Habitacional Popular entrega sus últimas viviendas en febrero de 1987 y, en este mismo mes, "fase dos" inicia su última obra. Aunque los rezagos ya señalados permiten prever la prolongación de estos programas en atención a los damnificados hasta mediados del año en curso, por lo menos, no es arriesgado afirmar que la política de reconstrucción habitacional, en lo esencial, ya se acabó.

Las consideraciones planteadas arriba trataron de identificar los aspectos de la política de reconstrucción habitacional que dejarán huellas permanentes en la política de vivienda en México. En este sentido, y al igual que el desastre que la provocó, el programa Renovación Habitacional Popular dejará indudablemente señas visibles en el centro de la ciudad. Los "condominios vecinales" y la población que los ocupa seguirán atestiguando esta experiencia excepcional de regeneración urbana: una regeneración que ha podido beneficiar a los habitantes originales gracias a la aplicación de criterios flexibles, tanto en el aspecto de la normatividad de la construcción y planeación como en las condiciones financieras. Desafortunadamente, sin embargo, y con alguna que otra excepción anotada en el presente ensayo, la política habitacional general del país parece regresar a sus tendencias de siempre.

${ }^{2}$ Un buen número de beneficiarios del programa "fase dos" ha logrado negociar la exención del pago inicial de $10 \%$, mismo que es cubierto por donativos de diversos organismos de beneficiencia, nacionales e internacionales: 


\section{Bibliografía}

CEPAL (1985), Daños causados por el movimiento telúrico y sus repercusiones sobre la economía, México, CEPAL.

CME (1985a), Informe (19 de septiembre a 19 de octubre de 1985), Comisión Metropolitana de Emergencia, suplemento de los principales periódicos capitalinos, México.

CME (1985b), Informe final, suplemento de los principales periódicos capitalinos, México, 20 de diciembre.

Coplamar (1982), Necesidades esenciales en México, vol. 3: vivienda, Coordinación General del Plan Regional de Zonas Deprimidas y grupos Marginados, México Siglo XXI.

Coulomb Bosc, René (1985), Transformaciones del espacio central y problemática habitacional, México, UAM-Azcapotzalco, depto. de Sociología.

DDF (1984a), Programa de reordenación urbana y protección ecológica del Distrito Federal, México, Departamento del Distrito Federal.

DDF (1984b), Sistema de normas de planificación urbana para el Distrito Federal, México, Departamento del Distrito Federal, Dirección General de Planificación.

Fonhapo (1986), Las consecuencias del sismo de septiembre de 1985 en la ciudad de México, particularmente en la vivienda, México, Fideicomiso del Fondo Nacional de Habitaciones Populares, Coordinación de Asesores.

López Monjardin, Adriana y Carolina Verduzco Ríos (1986), "Vivienda popular y reconstrucción", en Cuadernos Políticos, 45, enero-marzo, de 1986, pp. 25-37.

RHP (1986), Programa operativo, Renovación Habitacional Popular en el Distrito Federal, México.

Rodríguez Sáiz, Juan Manuel (1986), "Organizaciones populares y lucha política", en Cuadernos Políticos, 45, enero-marzo de 1986, pp. 38-55.

Sedue (1985), Programa nacional de vivienda 1985, México, Secretaría de Desarrollo Urbano y Ecología.

Ziccardi, Alicia (1986), Política de vivienda para un espacio destruido, México, IISUAM. 